\title{
PET/CT Alignment for Small Animal Scanners based on Capillary Detection
}

\author{
Alexia Rodríguez-Ruano, Javier Pascau, Member, IEEE, Judit Chamorro, Alejandro Sisniega, Member, IEEE, \\ Verónica García-Vázquez, Ángel Udías, Juan José Vaquero, Senior Member, IEEE, Manuel Desco
}

\begin{abstract}
Small animal PET/CT devices provide anatomical and molecular imaging at the same time, enabling the joint visualization and analysis of both modalities. An accurate PET/CT alignment is required to correctly interpret these studies. A proper calibration procedure is essential for small animal imaging, since resolution is much higher than in human devices. This work presents an alignment phantom and a method that enable a reliable and replicable measurement of the geometrical relationship between PET and CT modules. The phantom can be built with laboratory materials, and is used to estimate the rigid spatial transformation that aligns both modalities. It consists of three glass capillaries located in noncoplanar triangular geometry and filled with FDG, so they are easily identified in both modalities. The method is based on automatic line detection and localization of the corresponding points between the lines on both modalities, which allows calculating the rigid alignment parameters. Different geometric configurations of the phantom (i.e. different angles and distances between capillaries) were tested to assess the repeatability of the calculations. To measure the alignment precision achieved, we attached two additional sodium point sources to the phantom, which were neglected in the registration process. Our results show that the accuracy of the alignment estimation, measured as average misalignment of the Na sources, is below half the PET resolution. The alternative settings for the phantom layout did not affect this result, indicating the low dependency of the alignment calculated with the actual phantom layout. Our approach allows measuring the PET/CT transformation parameters using an in-house built phantom and with low computational effort and high accuracy, demonstrating that the proposed phantom is suitable for alignment calibration of dual modality systems on a real environment.
\end{abstract}

\section{INTRODUCTION}

PET and CT is a useful combination in molecular imaging. providing not only anatomical support to functional information in joint visualization but also making possible other applications, such as attenuation correction for improving PET image reconstruction process $[1,2]$. Furthermore, several clinical studies have shown that PET and CT, when used together, increase the diagnostic accuracy [3, 4]. In PET/CT devices, instead of using two different

Manuscript received November 14,2008 . This work is partially funded by the CD-TEAM Project, CENIT Program, Spanish Ministerio de Industria and with grants from the Ministerio de Educacion $\mathrm{y}$ Ciencia, projects TEC2007-64731 and TEC2008-06715-C02-01

A Rodríguez-Ruano, J Pascau, J Chamorro, A Sisniega, V GarcíaVázquez, JJ Vaquero and M Desco are with Unidad de Medicina y Cirugía Experimental Hospital General Universitario Gregorio Marañón, Madrid, Spain (e-mail: desco@mce.hggm.es).

A. Udias is with Departamento de Estadística e Investigación Operativa Universidad Rey Juan Carlos, 28943 Fuenlabrada, Spain. instruments, PET and CT systems are axially integrated and aligned in such a way that the sample can be transferred from one system gantry to the other automatically, reducing the risk of misalignments. However, the two axially displaced fields of view (FOVs) can be mechanically aligned only to a certain degree and the residual errors can impair sub-millimeter registrations. Since resolution is much higher than in human devices, molecular imaging of rats and mice requires precise registration of anatomical and functional imaging data [5].Therefore, PET/CT systems for small animal imaging require a calibration procedure to calculate accurately these residual misalignments.

There are several registration techniques to match intermodality images [6], but in order to automatically align different devices, complex and difficult to manufacture phantoms are sometimes needed. Chow et alt. [7] designed a three dimensional phantom with 1288 lines that is used with an automated registration method to estimate the transformation matrix. In contrast, other authors make the complexity lay on the registration method. Jan et alt [8] used a three line source phantom, where the determined pairs of homologous points between $\mathrm{CT}$ and PET images by measuring the distance between the line sources through 13 slices and then establishing correspondences. Methods based on landmarks distributions along the field of view have also been described $[9,10,11]$. Despite being simple and requiring minimal user interaction, the problem of these markers methods is that the user must place the point sources uniformly throughout the FOV. According to [12], target registration error (TRE), that is, registration error at some point of interest (target), depends both on fiducial configuration and on the target position. So the TRE will be lower as the target is nearer to the fiducial's centroid, the fiducials are more separate and they are distributed along different axis. Consequently, using fiducial markers to estimate the spatial transformation matrix may produce suboptimal results if the user is not concerned with its positioning. One solution to this problem is to provide the user with a rigid structure with which to place the point sources conveniently [9].

The challenge in alignment of PET/CT systems is to design a phantom that allows reliable misalignment calculations, with low computational cost and no user dependence. It should also be suitable for alignment calibration of dual modality systems in an end-user environment, since according to [9] the system needs to be calibrated regularly. 
This work presents an alignment phantom and a method that allow a reliable and replicable measurement of the geometrical relationship between PET and CT modules. We have designed a three-dimensional phantom that can be built with standard laboratory materials. The method is based on automatic line detection and localization of corresponding points within the lines from both modalities, allowing the rigid alignment to be calculated.

\section{MATERIAL AND METHODS}

\section{A. Phantom description}

The phantom was intended to be easy to manufacture in a final user environment, and to provide high accuracy and low dependence with its position within the field of view.

For this purpose, we have devised a phantom consisting on three non-coplanar glass capillaries filled with FDG, so as it results visible in both modalities. These capillaries are placed with a triangular geometry in the plan view, but each one of them located at a different height (Fig. 1). The capillaries used are micropipettes BLAUBRAND intraMARK Cat. No. $708709(10 \mu 1 / 1.24 \mathrm{~mm}$ external diameter). A low density foam structure is employed to separate the capillaries, in order to provide large density differences between glass capillaries and background material.

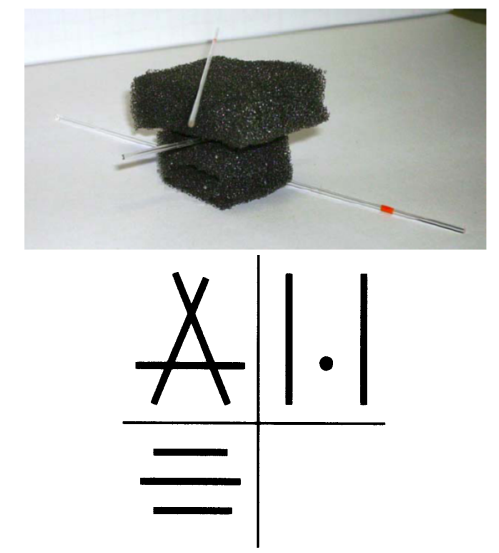

Fig. 1: Capillary phantom: photograph (top) and orthographic view (bottom)

\section{B. Image Acquisition}

A small animal PET/CT system (Argus/CT, Suinsa Medical Systems) has been used for the acquisition of CT and PET images $[13,14]$. The proposed methods were applied to 5 pairs of images, with different geometric configurations of the capillaries. Fig 2 depicts a render view of the phantom on both modalities, and the fused datasets.

With the purpose of evaluating the alignment precision against the layout of the capillaries, the geometric relations (angles and distances) were modified between acquisitions. To determine registration accuracy, two additional ${ }^{22} \mathrm{Na}$ point sources were acquired simultaneously with each study, but did not have effect on registration process.
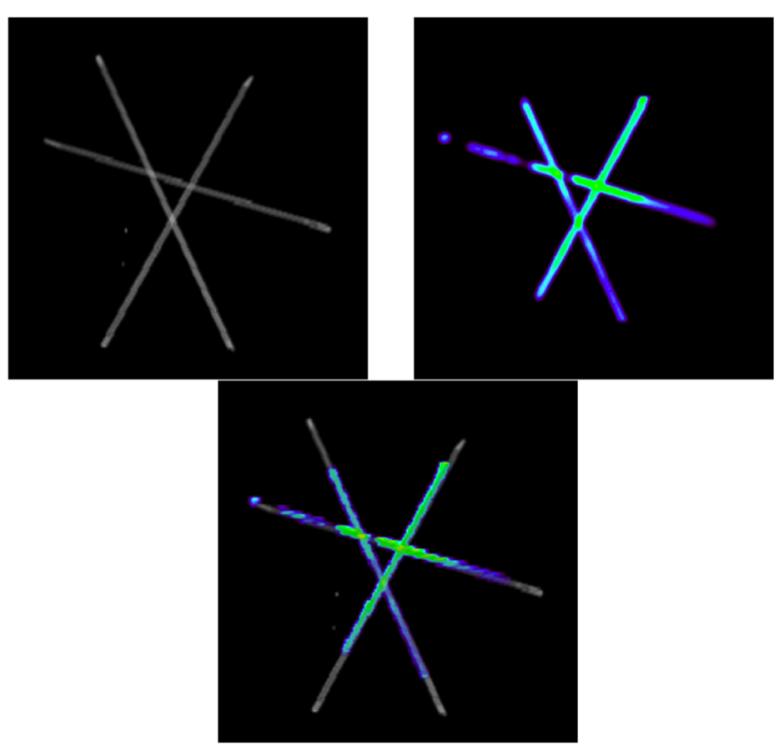

Fig. 2. Image of the phantom a) CT b) PET c) Fused datasets

\section{Registration Method}

A 3-D rigid transformation (including only translations and rotations) has to be found for each PET/CT image pair. This transformation will be obtained by locating pairs of homologous points along the capillaries on both modalities and aligning them. The procedure to uniquely define these landmarks is based on locating the closest point from one line to another within the line set. The procedure can be split into four steps:

\section{1) Background Thresholding and Region Labeling}

Once the phantom has been imaged, the first step is to detect the lines that describe the capillaries in the images. Initially, the images are reduced to point sets that correspond with the capillaries by thresholding the background and labeling the resulting regions as:

$\mathrm{X}_{\mathrm{n}}=$ [points on CT image corresponding to capillary $\mathrm{n}$ ]

$\mathrm{Y}_{\mathrm{n}}=$ [points on PET image corresponding to capillary $\mathrm{n}$ ] $\mathrm{n}=1,2,3$

2) Line Detection

Principal Component Analysis (PCA) is applied over each distribution of points $X_{n}$ and $Y_{n}$, to detect the lines that describe the position and orientation of each of the capillaries [15].

Here, $\mathrm{X}_{\mathrm{n}}=\left(\mathrm{a}_{\mathrm{n}}, \vec{u}_{n}\right)$ is the line related to capillary $\mathrm{n}$ on the CT image, represented by one of its points $a_{n}$ and its direction vector $\vec{u}_{n}$. Similarly, in PET image, $\mathrm{Yn}=\left(\mathrm{bn}, \vec{v}_{n}\right)$.

At this point, each data set has been reduced to three line equations.

3) Homologous Points Identification on $X_{n}$ and $Y_{n}$

As mentioned before, this method approximates the best matching of both line sets by matching corresponding points pairs extracted from the line sets in order to calculate the transformation matrix. 
The procedure to define these landmark pairs is based on locating the closest point from one line to another within the line set. The common perpendicular between two lines within the same line set defines the closest points between those lines, which are uniquely defined in both modalities (Fig. 4). The result of this process is six pairs of homologous landmarks. For instance, the closest point to $\mathrm{X} 2$ on $\mathrm{X} 1$ corresponds with the closest point to Y2 on Y1.
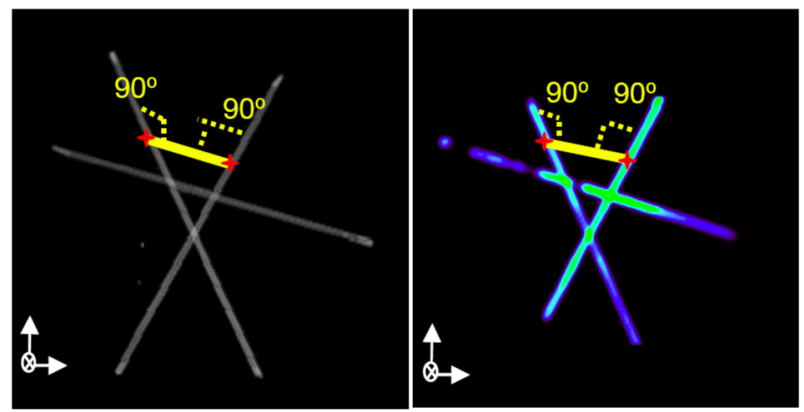

Fig 3: Homologous points identification

\section{4) Transformation Matrix Estimation}

These landmarks are used to calculate the transformation that aligns the images by solving the Least Squares problem using the approach proposed in [16]. The final PET/CT alignment parameters (translation and rotation) can be derived from the transformation matrix obtained.

\section{RESULTS}

The alignment accuracy was estimated using the ${ }^{22} \mathrm{Na}$ point sources included on the acquisition of every phantom. The average mean squared error at these references was $0.477 \mathrm{~mm}$ (Table I).

Three different geometric configurations of the capillaries (i.e. different angles and distances between capillaries) were tested to assess the repeatability of the calculations. The alignment accuracy was estimated using the validation described previously. The transformation calculated through both methods was applied to this phantom to measure the error at each ${ }^{22} \mathrm{Na}$ point source (note that the sources were neglected during the registration process). The average mean squared error at these references was $0.477 \mathrm{~mm}$ (Table I).

TABLE I

MEAN SQUARE ERROR

\begin{tabular}{l|c} 
& \multicolumn{2}{c}{ MEAN SQUARE ERROR } \\
& $\begin{array}{c}\text { Error on Na source } \\
\text { (mm) }\end{array}$ \\
\hline Configuration 1 & 0.269 \\
Configuration 2. Repetition 1 & 0.492 \\
Configuration 2. Repetition 2 & 0.538 \\
Configuration 3. Repetition 1 & 0.493 \\
Configuration 3. Repetition 2 & 0.440 \\
\hline Mean & $\mathbf{0 . 4 4 7}$ \\
\hline
\end{tabular}

\section{CONCLUSIONS}

Results demonstrate that the method and the phantom presented can estimate the PET/CT alignment in a dualmodality system, with an accuracy bellow half the PET resolution. The alternative settings for the phantom layout did not affect the results noticeably, indicating the low dependency of the alignment calculated with the actual phantom layout.

Unlike previous methods that rely on complex phantoms or intensive user intervention, our method automatically calculates the misalignment between FOVs and requires the use of a simple phantom for calibration. In contrast with algorithms based on fiducial landmarks, the accuracy of the method does not depend on the geometric configuration of the phantom. As a result, target registration error is not dependent on the positioning of the phantom. This will allow different users to build their own phantom in order to calibrate the PET/CT.

Our approach allows measuring the PET/CT transformation parameters using a simple phantom, with low computational effort and high accuracy, suggesting that the proposed phantom is suitable for alignment calibration of dual modality systems in an end-user environment.

\section{REFERENCES}

[1] C. Burger, G. Goerres, S. Schoenes, A. Buck, A. H. Lonn, and G. K. Von Schulthess, "PET attenuation coefficients from CT images: experimental evaluation of the transformation of CT into PET $511-\mathrm{keV}$ attenuation coefficients," Eur J Nucl Med Mol Imaging, vol. 29, pp. 922-7, Jul 2002

[2] P. E. Kinahan, D. W. Townsend, T. Beyer, and D. Sashin, "Attenuation correction for a combined 3D PET/CT scanner," Med Phys, vol. 25, pp. 2046-53, Oct 1998

[3] M. J. Dong, K. Zhao, X. T. Lin, J. Zhao, L. X. Ruan, and Z. F. Liu, "Role of fluorodeoxyglucose-PET versus fluorodeoxyglucosePET/computed tomography in detection of unknown primary tumor: a meta-analysis of the literature," Nucl Med Commun, vol. 29, pp. 791802, Sep 2008.

[4] S. J. Rosenbaum, T. Lind, G. Antoch, and A. Bockisch, "False-positive FDG PET uptake--the role of PET/CT," Eur Radiol, vol. 16, pp. 105465, May 2006.

[5] J. J. Vaquero, M. Desco, J. Pascau, A. Santos, I. Lee, J. Seidel, and M. V. Green, "PET, CT, and MR Image Registration of the Rat Brain and Skull," IEEE T Nucl Sci, vol. 48, pp. 1440-1445, 2001.

[6] D. L. Hill, P. G. Batchelor, M. Holden, and D. J. Hawkes, "Medical image registration," Phys Med Biol, vol. 46, pp. R1-45, Mar 2001

[7] P. L. Chow, D. B. Stout, E. Komisopoulou, and A. F. Chatziioannou, "A method of image registration for small animal, multi-modality imaging," Phys Med Biol, vol. 51, pp. 379-90, Jan 212006.

[8] M.-L. Jan, Y.-C. Ni, K.-W. Chen, H.-C. Liang, K.-S. Chuang, and Y.$\mathrm{K}$. Fu, "A combined micro-PET/CT scanner for small animal imaging," Nuclear Instruments and Methods in Physics Research Section A: Accelerators, Spectrometers, Detectors and Associated Equipment, vol 569, pp. 314-318, 2006

[9] R. Gregory, M. Partridge, and M. A. Flower, "Performance Evaluation of the Philips \&\#8220;Gemini\&\#8221; PET/CT System," Nuclear Science, ItFE Transactions on, vol. 53, pp. 93-101, 2006.

[10] H. Liang, Y. Yang, K. Yang, Y. Wu, J. M. Boone, and S. R. Cherry, "A microPET/CT system for in vivo small animal imaging," Phys Med Bıol, vol. 52, pp. 3881-94, Jul 72007.

[11] J. J. Vaquero, J. Pascau, M. Abella, A. Sisniega, E. Lage, and M Desco, "Field of view alignment on a multimodality PET/CT scanner for small animals," in 2007 leee Nuclear Science Symposium ('onference Record, Vols l-1l New York: Ieee, 2007, pp. 2868-2869. 
[12] J. M. Fitzpatrick and J. B. West, "The distribution of target registration error in rigid-body point-based registration," Medical Imaging, IEFE: Transactions on, vol. 20, pp. 917-927, 2001

[13] J. J. Vaquero, S. Redondo, E. Lage, M. Abella, A. Sisniega, G. Tapias, M. L. Soto-Montenegro, and M. Desco, "Assessment of a New HighPerformance Small-Animal X-Ray Tomograph," IEEE T Nucl Sci, vol. 55, pp. 898-905, 2008.

[14] Y. Wang, J. Seidel, B. M. W. Tsui, J. J. Vaquero, and M. G. Pomper, "Performance Evaluation of the GE Healthcare eXplore VISTA DualRing Small-Animal PET Scanner," $J$ Nucl Med, vol. 47, pp. 18911900, 2006.

[15] R. Duda, P. Hart, and D. Stork, Pattern (lassificution (2nd Edition) Wiley-Interscience, 2000

[16] K. S. Arun, T. S. Huang, and S. D. Blostein, "Least-squares fitting of two 3-D point sets," IEFE Trans. Pattern Anal. Mach. Intell., vol. 9, pp. $698-700,1987$. 